\title{
RICCI SOLITONS ON NEARLY KENMOTSU MANIFOLDS WITH SEMI-SYMMETRIC METRIC CONNECTION
}

\author{
Gülhan Ayar ${ }^{\mathrm{a} *}$, Dilek Demirhan ${ }^{\mathrm{a}}$ \\ ${ }^{a}$ Karamanoglu Mehmetbey University, Department of Mathematics, Karaman, Turkey \\ gulhanayar@kmu.edu.tr (*corresponding author),dilekdemirhan11@gmail.com
}

\begin{abstract}
In this work, we give some basic informations about Ricci solitons on nearly Kenmotsu manifolds and some structures on this manifolds satisfying semi-symmetric metric connection. Then we consider some important results and theorems of Ricci solitons on Ricci-recurrent and $\Phi$-recurrent nearly Kenmotsu manifolds with semi-symmetric metric connection. Also final part of the present paper, we study Ricci solitons on quasi-projectively flat nearly Kenmotsu manifolds with semi-symmetric metric connection.
\end{abstract}

Keywords: Nearly Kenmotsu manifold, Ricci soliton, semi-symmetric metric connection

\section{Introduction}

Although Ricci flows represent the study of physical science, it has recently become an important subject of study in mathematics, especially in differential geometry [1]. Most of these studies are composed of Ricci solitons on various manifolds. Especially considering the history of contact structures, Ricci flow has been a starting point for us in the formation of contact manifolds. Ricci flows were first introduced by Hamilton [2] in 1982. If we refer about the Ricci flow equation on a compact Riemannian manifold $M^{2 n+1}$, we can give the following formula with the Riemannian metric $g$ by:

$$
\frac{\partial g}{\partial t}=-2 \operatorname{Ricg}
$$

such that Ricg is defined as Ricci curvature tensor and $t$ is time.

By the way on a Riemannian manifold $\left(M^{2 n+1}, g\right)$, a Ricci soliton is a soliton defined by Hamilton [4] similar to the Ricci flow and moves only with a one-parameter of the difeomorphism family. Also it is defined by the following relation 
$\left(\mathcal{L}_{V} g\right)(X, Y)+2 S(X, Y)+2 \lambda g(X, Y)=0$

such that $\mathrm{S}$ is the Ricci tensor associated to $g, L_{V}$ is the Lie derivative operator along the vector field and $\lambda$ is a real scalar [5].

It is well known that nearly Kenmotsu manifolds can be characterized through their Levi-Civita connection by

$$
\left(\nabla_{X} \phi\right) Y+\left(\nabla_{Y} \phi\right) X=-\eta(Y) \phi X-\eta(X) \phi Y
$$

for any vector fields $X$ and $Y$ [6].

Moreover if $M^{2 n+1}$ satisfies the following equation

$$
\left(\nabla_{X} \phi\right) Y=-g(\phi X, Y) \xi-\eta(Y) \phi X
$$

then it is called Kenmotsu manifold [7].

The notion of semi-symmetric manifold is defined by

$$
R(X, Y) \cdot R=0,
$$

for all vector fields $X, Y$ on $M^{2 n+1}$, where $R(X, Y)$ function as a derivation on $R$ [8]. At a point $p \in M^{2 n+1}$, curvature tensor of $\left(M^{2 n+1}, g\right), R_{p}$, is called "semi-symmetric space", when it is same as the curvature tensor of a symmetric space [6].

Ricci soliton and gradient Ricci soliton and also $\eta$ - Ricci solitons on nearly Kenmotsu manifolds have been studied before $[14,15]$. In this paper we have studied Ricci solitons on nearly Kenmotsu manifolds under semi-symmetric conditions. We give certain properties of Ricci solitons on a nearly Kenmotsu manifold with semi-symmetric metric connection. In section 2, basic definitions and informations about Ricci solitons on nearly Kenmotsu manifolds have been given. In the next section, we give some curvature identities about curvature tensor of Ricci solitons on nearly Kenmotsu manifolds with semi-symmetric metric connection. In sections 4 and 5, we find some basic results and theorems on Ricci-recurrent and $\phi$-recurrent nearly Kenmotsu manifolds with semi-symmetric metric connection. Final part of the present paper deals with the study of Ricci solitons on quasi-projectively flat nearly Kenmotsu manifolds with semi-symmetric metric connection.

\section{Preliminaries}

Throughout the paper, all objects are to be regard as $C^{\infty}$ class manifolds and we accept $X, Y, Z, W \in \chi\left(M^{2 n+1}\right)$ as vector fields on $M^{2 n+1}$.

Let $\left(M^{2 n+1}, \phi, \xi, \eta, g\right)$ be an $(2 n+1)$ - dimensional differentiable almost contact Riemannian manifold with the compatible geometric structures on, such that $\phi$ is a $(1,1)$-tensor field, $\xi$ is a characteristic vector field, $\eta$ is a 1 -form and $g$ is a Riemannian metric. With this concept, the structure shown in the form of is called almost contact metric structure on $M^{2 n+1}$. Meanwhile $(\phi, \xi, \eta, g)$ structure satisfies the following conditions [9] 
$\eta(\xi)=1$

$\eta \circ \phi=0$,

$\phi^{2} X=-X+\eta(X) \xi$

$g(\phi X, \phi Y)=g(X, Y)-\eta(X) \eta(Y)$,

where $X, Y \in \chi\left(M^{2 n+1}\right)$.

In addition to these features, an almost contact metric structure with $(\phi, \xi, \eta, g)$ satisfies the following conditions:

$\phi(\xi)=0$,

$\eta(\xi)=1$

$\eta(\phi X)=0$,

$\eta(X)=g(X, \xi)$,

$g(X, \phi Y)+g(Y, \phi X)=0$.

Moreover, satisfying

$$
d \eta(X, Y)=g(X, \phi Y)
$$

condition, a Kenmotsu manifold $\left(M^{2 n+1}, \phi, \xi, \eta, g\right)$ is called almost contact metric manifold. In 1972 Kenmotsu manifolds have been studied by Kenmotsu [7].

An almost contact manifold $\left(M^{2 n+1}, \phi, \xi, \eta, g\right)$ is called nearly Kenmotsu manifold by Shukla with the following relation:

$\left(\nabla_{X} \phi\right) Y+\left(\nabla_{Y} \phi\right) X=-\eta(Y) \phi X-\eta(X) \phi Y$,

where $\nabla$ is Levi-Civita connection of $g$ [10].

As a result, it is understood that every Kenmotsu manifold is nearly Kenmotsu manifold but converse is not true [11].

In addition, we can give the following relations on an $(2 n+1)-$ dimensional nearly Kenmotsu manifold $M^{2 n+1}$ without proofs that we will use throughout this paper [11]:

$$
\begin{aligned}
& \nabla_{X} \xi=X-\eta(X) \xi, \nabla_{\xi} \xi=0 \\
& \left(\left(\nabla_{X} \phi\right)(Y)=g(X, Y)-\eta(X) \eta(Y),\right. \\
& R(X, Y) \xi=\eta(X) Y-\eta(Y) X \\
& R(\xi, Y) Z=-g(Y, Z) \xi+\eta(Z) Y, \\
& R(\xi, Y) \xi=-R(Y, \xi) \xi=X-\eta(X) \xi \\
& \eta(R(X, Y) Z)=g(X, Z) \eta(Y)-g(Y, Z) \eta(X), \\
& S(X, \xi)=-2 Y \eta(X),
\end{aligned}
$$


$Q X=-2 Y X$

$Q \xi=-2 Y \xi$

$S(\phi X, \phi Y)=S(X, Y)+2 Y \eta(X) \eta(Y)$

$R(\phi X, Y, Z, W)+R(X, \phi Y, Z, W)+R(X, Y, \phi Z, W)+R(X, Y, Z, \phi W)=0$,

$R(\xi, Y, Z, W)=\eta(Z) g(Y, W)-\eta(W) g(Y, Z)$,

$R(\phi X, \phi Y, Z, W)=R(X, Y, \phi Z, \phi W)$,

$R(\phi X, \phi Y, \phi Z, \phi W)=R(X, Y, Z, W)-\eta(X) R(\xi, Y, Z, W)+\eta(Y) R(\xi, X, Z, W)$

for any vector fields $X, Y, Z, \in \chi\left(M^{2 n+1}\right)$.

In the above equations, $R$ is both $(1,3)$ type Riemannian curvature tensor and $(0,4)$ type Riemannian curvature tensor defined as $R(X, Y, Z, W)=g(R(X, Y) Z, W)$.

$S$ is the Ricci tensor of type $(0,2)$ defined as $S(X, Y)=g(Q X, Y)$ depending on the Ricci operator $Q$

Now we will give some definitions that we will use for our results later:

Definition 2.1 Let $M$ be an $2 n+1$ dimensional nearly Kenmotsu manifold. A connection $\tilde{\nabla}$ on $M$ is called semi-symmetric connection if its torsion tensor

$T(X, Y)=\tilde{\nabla}_{X} Y-\tilde{\nabla}_{Y} X-[X, Y]$

satisfies

$T(X, Y)=\eta(Y) X-\eta(X) Y$,

besides, a semi-symmetric connection with

$\left(\tilde{\nabla}_{X} g\right)(Y, Z)=g\left(\tilde{\nabla}_{X} Y, Z\right)+g\left(Y, \tilde{\nabla}_{X} Z\right)$

condition is called semi-symmetric metric connection.

Definition 2.2 Let $M^{2 n+1}$ be an $2 n+1$ dimensional nearly Kenmotsu manifold and $\tilde{\nabla}$ be the Levi-Civita connection on $M^{2 n+1}$. Then $\tilde{\nabla}$

$\tilde{\nabla}_{X} Y=\nabla_{X} Y+\eta(Y) X-g(X, Y) \xi$

is the semi-symmetric metric connection on $M^{2 n+1}$.

In the above definitions curvature tensor, the Ricci tensor, the Ricci operator and the scalar curvature are defined as $\tilde{R}, \tilde{S}, \tilde{Q}$ and $\tilde{r}$ respectively on a nearly Kenmotsu manifold with semisymmetric metric connection $\tilde{\nabla}$ (Linear connection). 
Similarly, we give the curvature tensor, the Ricci tensor, the Ricci operator and the scalar curvature as $R, S, Q$ and $r$ respectively of a nearly Kenmotsu manifold with semi-symmetric metric connection $\nabla$ (Levi-Civita connection) [12].

Lemma 2.3 Let $M^{2 n+1}$ be an $2 n+1$ dimensional nearly Kenmotsu manifold with semisymmetric metric connection. If the manifold satisfies the connection (1) then we have

$\tilde{S}(X, Y)=-(\lambda+1) g(X, Y)+\eta(X) \eta(Y)$.

The above equation yields

$$
\begin{aligned}
& \tilde{Q} X=-(\lambda+1) X+\eta(X) \xi \\
& \tilde{S}(X, \xi)=-\lambda \eta(X) \\
& \tilde{r}=-\lambda(2 n+1)-2 n . \\
& \left(\tilde{\nabla}_{W} \tilde{S}\right)(Y, \xi)=\tilde{\nabla}_{W} \tilde{S}(Y, \xi)-\tilde{S}\left(\tilde{\nabla}_{W} Y, \xi\right)-\tilde{S}\left(Y, \tilde{\nabla}_{W} \xi\right) .
\end{aligned}
$$

By the equation (1) and (6) one can easly show.

Definition 2.4 On a $2 n+1$ dimensional nearly Kenmotsu manifold with semi-symmetric metric connection, some basic curvature properties as follows [12]:

$\tilde{R}(X, N) Z=\tilde{\nabla}_{X} \tilde{\nabla}_{N} Z-\tilde{\nabla}_{N} \tilde{\nabla}_{X} Z-\tilde{\nabla}_{[X, N]} Z$,

$\tilde{R}(X, Y) Z=R(X, Y) Z+3[g(X, Z) Y-g(Y, Z) X+2[g(Y, Z) \eta(X)-g(X, Z) \eta(Y)] \xi+$ $2[\eta(Y) X-\eta(X) Y] \eta(Z)$,

where

$$
\begin{array}{r}
R(X, N) Z=\nabla_{X} \nabla_{N} Z-\nabla_{N} \nabla_{X} Z-\nabla_{[X, N]} Z, \\
\tilde{S}(Y, Z)=S(Y, Z)+(2-6 n) g(Y, Z)+2(2 n-1) \eta(Y) \eta(Z) .
\end{array}
$$

Besides, Ricci soliton on a nearly Kenmotsu manifold with semi-symmetric metric connection satisfies the following equations [12]:

$$
\begin{aligned}
& \tilde{S}(Y, \xi)=-4 n \eta(Y), \\
& \tilde{R}(X, Y) \xi=2[\eta(X) Y-\eta(Y) X], \\
& \tilde{R}(\xi, Y) Z=2[-g(Y, Z) \xi+\eta(Z) Y], \\
& \tilde{R}(\xi, Y) \xi=2[Y-\eta(Y) \xi], \\
& \tilde{R}(X, Y) Z=-\tilde{R}(Y, X) Z, \quad \tilde{R}(\xi, Y) \xi=-\tilde{R}(Y, \xi) \xi,
\end{aligned}
$$


$\tilde{Q} Y=-4 n Y, \quad \tilde{Q} \xi=-4 n \xi$,

$\tilde{S}(\phi Y, \phi Z)=S(Y, Z)+(2-6 n) g(Y, Z)+2(2 n-2) \eta(Y) \eta(Z)$,

$\tilde{r}=r-12 n^{2}+2 n$

$\tilde{\nabla}_{X} \xi=2(X-\eta(X) \xi)$,

$\eta(\tilde{R}(X, Y) Z)=2[g(X, Z) \eta(Y)-g(Y, Z) \eta(X)]$.

From equation (30), we get

$\tilde{R}(Y, Z) X=R(Y, Z) X+3[g(Y, X) Z-g(Z, X) Y]+2[g(Z, X) \eta(Y)-g(Y, X) \eta(Z)] \xi+$ $2[\eta(Z) Y-\eta(Y) Z] \eta(X)$,

$\tilde{R}(Z, X) Y=R(Z, X) Y+3[g(Z, Y) X-g(X, Y) Z]+2[g(X, Y) \eta(Z)-g(Z, Y) \eta(X)] \xi+$ $2[\eta(X) Z-\eta(Z) X] \eta(Y)$.

From equations (30), (42) and (43), we get

$\tilde{R}(X, Y) Z+\tilde{R}(Y, Z) X+\tilde{R}(Z, X) Y=0$.

\section{Ricci solitons of Ricci-recurrent nearly Kenmotsu manifolds with semi- symmetric metric connection}

Definition 3.1 A nearly Kenmotsu manifold with semi-symmetric metric connection is said to be Ricci-recurrent manifold if there exists a non-zero 1 -form $A$ such that

$\left(\tilde{\nabla}_{W} \tilde{S}\right)(Y, Z)=A(W) \tilde{S}(Y, Z)$.

Theorem 3.2 Ricci soliton on Ricci-recurrent nearly Kenmotsu manifold $M^{2 n+1}$ with semisymmetric metric connection by the $1-$ form $A$ is

- expanding if $A(\xi)<2$,

- steady if $A(\xi)=2$,

- shrinking if $A(\xi)>2$.

Proof. Replacing for $U=\xi$ in (45), we get

$\left(\tilde{\nabla}_{W} \tilde{S}\right)(Y, \xi)=A(W) \tilde{S}(Y, \xi)$.

Using (12) and (6) in (28), we obtain

$\left(\tilde{\nabla}_{W} \tilde{S}\right)(Y, \xi)=-8 n g(Y, W)-2 \tilde{S}(Y, W)$.

In view of (46) and (47), we have

$\tilde{S}(Y, W)=-4 n g(Y, W)+2 n \eta(Y) A(W)$. 
Taking $Y=\xi$ in (48), we get

$\tilde{S}(\xi, W)=-4 n \eta(W)+2 n A(W)$.

Applying (26), (49) reduces to

$2 n A(W)=(4 n-\lambda) \eta(W)$.

Using (26) in (50) and for $W=\xi$, we obtain

$\lambda=2 n[2-A(\xi)]$

\section{Ricci soliton on $\phi$-recurrent nearly Kenmotsu manifolds with semi- symmetric metric connection}

Definition 4.1 A nearly Kenmotsu manifold with semi-symmetric metric connec-tion is said to be a $\phi$-recurrent manifold with if non-zero 1 -form $A$ satisfies the following condition [13]

$\phi^{2}\left(\left(\tilde{\nabla}_{W} \tilde{R}\right)(X, Y) Z\right)=A(W) \tilde{R}(X, Y) Z$

for arbitrary vector fields $X, Y, Z, W$.

Theorem 4.2 Ricci soliton on a $\phi$-recurrent nearly Kenmotsu manifold $M^{2 n+1}$ with semisymmetric metric connection with the 1 -form $A$ is

- $\quad$ expanding if $A(\xi)>-2$,

- steady if $A(\xi)=-2$,

- shrinking if $A(\xi)<-2$.

Proof. Let us consider a $\phi$-recurrent nearly Kenmotsu manifold with semi-symmetric metric connection. By virtue of (2) and (52), we have

$-\left(\tilde{\nabla}_{W} \tilde{R}\right)(X, Y) Z+\eta\left(\left(\tilde{\nabla}_{W} \tilde{R}\right)(X, Y) Z\right) \xi=A(W) \tilde{R}(X, Y) Z$

Contracting (53) with $Z$, we obtain

$-g\left(\left(\tilde{\nabla}_{W} \tilde{R}\right)(X, Y) Z, Z\right)+\eta\left(\left(\tilde{\nabla}_{W} \tilde{R}\right)(X, Y) Z\right) \eta(Z)=A(W) g(\tilde{R}(X, Y) Z, Z)$.

Let $e_{i}(i=1,2, \ldots, 2 n+1)$, be an orthonormal basis of the tangent space at any point of the manifold. Taking $X=Z=e_{i}$ in (54) and taking summation over $i, 1 \leq i \leq 2 n+1$, we get

$-\left(\tilde{\nabla}_{W} \tilde{S}\right)(Y, Z)=A(W) \tilde{S}(Y, Z)$.

Replacing $Z$ by $\xi$ in (55) and using (12), we have

$-\left(\tilde{\nabla}_{W} \tilde{S}\right)(Y, \xi)=A(W) \tilde{S}(Y, \xi)$.

Using (12) and (6) in (28), we obtain 
$\left(\tilde{\nabla}_{W} \tilde{S}\right)(Y, \xi)=-8 n g(Y, W)-2 \tilde{S}(Y, W)$

In view of (56) and (57), we have

$\tilde{S}(Y, W)=-4 n g(Y, W)-2 n \eta(Y) A(W)$.

Taking $Y=\xi$ in (58), we get

$\tilde{S}(\xi, W)=-4 n \eta(W)-2 n A(W)$.

$2 n A(W)=(-4 n+\lambda) \eta(W)$

Using (26) in (60) and for $W=\xi$ we obtain

$\lambda=2 n[2+A(\xi)]$

\section{Ricci soliton on quasi-projectively flat nearly Kenmotsu manifolds with semi-symmetric metric connection}

Definition 5.1 Projective curvature tensor $\tilde{P}$ of type $(1,3)$ of an $(2 n+1)$ dimensional nearly Kenmotsu manifold with semi symmetric metric connection $\tilde{\nabla}$ is given by

$\tilde{P}(X, Y) Z=\tilde{R}(X, Y) Z-\frac{1}{2 n}[\tilde{S}(Y, Z) X-\tilde{S}(X, Z) Y]$

Ricci soliton on a nearly Kenmotsu manifold $M^{2 n+1}$ with semi-symmetric metric connection is said to be quasi-projectively if

$g(\tilde{P}(X, \phi Y) \phi Z, W)=0$.

where $\tilde{P}$ is the projective curvature tensor with respect to semi-symmetric metric connection.

Theorem 5.2 Ricci soliton of quasi-projectively flat nearly Kenmotsu manifold $M^{2 n+1}$ with semi-symmetric metric connection is

- expanding if $k<2$,

- $\quad$ steady if $k=2$,

- $\quad$ shrinking if $k>2$.

where $k \in \mathbb{R}$.

Proof. Taking inner product of equation (62) with $\mathrm{W}$ and using equations (30), (31) and (63), we get

$g(\tilde{R}(X, \phi Y) \phi Z, W)+3[g(X, \phi Z) g(\phi Y, W)-g(\phi Y, \phi Z) g(X, W)]+2 \eta(X) \eta(W) g(\phi Y, \phi Z)$

$=\frac{1}{2 n}[\tilde{S}(\phi Y, \phi Z) g(X, W)+(2-6 n) g(\phi Y, \phi Z) g(X, W-\tilde{S}(X, \phi Z) g(\phi Y, W)-(2-$ 6n) $g(X, \phi Z) g(\phi Y, W)]$. 
Let $\left\{e_{1}, e_{2}, \ldots, e_{2 n}, \xi\right\}$ be a local orthogonal basis of vector fields in $M^{2 n+1}$ then $\left\{\phi e_{1}, \phi e_{2}, \ldots, \phi e_{2 n}, \xi\right\}$ is also local orthonormal basis of $M^{2 n+1}$. Now putting the value $Y=$ $Z=e_{i}$ in the equation (64) and taking summation over $i, 1 \leq i \leq 2 n+1$, we get

$\tilde{S}(X, W)=\left(\frac{r}{2 n+1}+\frac{2(2 n-1)}{2 n+1}\right) g(X, W)-4 n \eta(X) \eta(W)+(2-6 n) g(X, W)+2(2 n-$ 1) $\eta(X) \eta(W)$.

Taking $W=\xi$ in the equation (65)

$\tilde{S}(X, \xi)=\left(\frac{r}{2 n+1}+\frac{2(2 n-1)}{2 n+1}\right) g(X, \xi)-4 n \eta(X) \eta(\xi)+(2-6 n) g(X, \xi)+2(2 n-$ 1) $\eta(X) \eta(\xi)$.

Applying (3), into (66) we have

$\tilde{S}(X, \xi)=\left(\frac{r}{2 n+1}+\frac{2(2 n-1)}{2 n+1}\right) \eta(X)-4 n \eta(X)+(2-6 n) \eta(X)+2(2 n-1) \eta(X)$.

Using (39) in (67) we get

$\tilde{S}(X, \xi)=\left(\frac{\tilde{r}}{2 n+1}-2\right) \eta(X)$

Applying (26), (68) reduces to

$-\lambda \eta(X)=\left(\frac{\tilde{r}}{2 n+1}-2\right) \eta(X)$

Taking $X=\xi$ in (69), we obtain

$\lambda=2-k$,

where $k$ is constant.

\section{References}

[1] Bejan, C.L., Crasmareanu, M., "Second order parallel tensors and Ricci solitons in 3dimensional normal paracontact geometry”, Ann. Glob. Anal. Geom. (2014), doi:10. 1007/s10455-014-9414-4.

[2] Hamilton, R.S., "Three manifolds with positive Ricci curvature”, Journal of Differential Geometry 17 (2) (1982) : 225-306.

[3] Hamilton, R.S., “The Ricci flow on surfaces”, Contemporary Mathematics 71 (1988) : 237-261.

[4] Hamilton, R.S., "The Ricci flow on surfaces. In: Mathematics and general relativity (Santa Cruz, CA, 1986)”, Contemp. Math. Amr. Math. Soc., Providence 71 (1988) : 237262.

[5] Nagaraja, H.G., Venu, K., “Ricci Solitons in Kenmotsu Manifold”, Journal of Informatics and Mathematical Sciences 8 (2016) : 29. 
[6] Oztürk, H., “On $\alpha$-Kenmotsu manifolds satisfying semi-symmetric conditions”, Konuralp Journal of Mathematics 5 (2017) : 192-193.

[7] Kenmotsu, K., “A class of contact Riemannian manifold”, Tohoko Math. J. 24 (1972) : 93-103.

[8] Nomizu, K., “On hypersurfaces satisfying a certain condition on the curvature tensor”, Tohoko Mat. J. 20 (1968) : 46-69.

[9] Blair, D.E., “Contact manifolds in Riemannian geometry”, Lecture Notes in Mathematics 509 (1976), Springer-Verlag, Berlin.

[10] Shukla, A., “Nearly trans-Sasakian manifolds”, Kuwait J. Sci. Eng. 23 (2) (1996) : 139144.

[11] Küpeli Erken, I., Piotr D., Murathan, C., “On the existence of proper nearly Kenmotsu manifolds”, Mediterr. J. Math. 13 (2016) : 4497-4507.

[12] Prasad, R., Kumar, S., Gautam, U.K., "On nearly Kenmotsu manifolds with semisymmetric metric connection”, Ganita 68 (1) (2018) : 133.

[13] De, U.C., “On $\phi$-recurrent Kenmotsu manifolds”, Turk J. Math. 33 (2009) : 17-25.

[14] Ayar, G., Yıldırım, M., " $\eta$-Ricci solitons on nearly Kenmotsu manifolds”, AsianEuropean Journal of Mathematics 12 (6) (2019) : 2040002.

[15] Ayar, G., Yıldırım M., "Ricci solitons and gradient Ricci solitons on nearly Kenmotsu manifolds”, Facta Universitatis (NIS) Ser. Math. Inform. 34 (3) (2019) : 503-510. 